\title{
RKHS approach for signal detection in rotation and scale space random fields
}

\author{
K. Shafie \\ Department of Applied Statistics and Research Methods, University of Northern Colorado, United States \\ khalil.shafie@unco.edu \\ Akbar Abravesh \\ Department of Applied Statistics, University of Mohaghegh Ardabili, Iran
}

Received 13 December 2014

Accepted 11 June 2016

\begin{abstract}
Two important papers of Worsley, Siegmund and coworkers consider rotation and scale space random fields for detecting signals in fMRI (functional magnetic resonance imaging) brain images. They use the global maxima of images for detection of a signal. In the current work, we utilize a reproducing kernel Hilbert space (RKHS) approach to show for both rotation and scale space random fields the global maximum of the image is indeed the likelihood ratio test statistic.
\end{abstract}

Keywords: Scale space random fields, rotation space random fields, reproducing kernel Hilbert space, likelihood ratio statistic.

2000 Mathematics Subject Classification: primary 62M40; 60G10; secondary 62M30

\section{Introduction}

For the past two decades, Gaussian random fields have been used in a variety of applications in astronomy, neural imaging and genetics to model images produced by modern sensor technologies. One of the main problems in these subject areas is searching for an activation or a signal in the images. Two papers of [1] and [2] consider rotation and scale space random fields for detecting signals in fMRI (functional magnetic resonance imaging) brain images. They use the global maxima of images for detection of a signal. For the scale space random field, [1] and for the rotation space random field, [2], without providing any proof, state that the global maximum, $X_{\max }$, is the likelihood ratio test static for testing the hypothesis of no signal. For the scale space random fields, [3] uses techniques of Gaussian measures on Hilbert spaces to present a proof. In the current work, we give another proof which works for both rotation and scale space random fields.

Reproducing kernel Hilbert spaces $(R K H S)$ arise in different areas of statistics, including statistical signal processing, nonparametric curve estimation, random measures, and limit theorems. Use of the RKHS approach for the problem of signal detection in Gaussian noise now is a classical method in signal processing literature. $[4,5]$ used the RKHS approach to find the likelihood ratio for detection problem in the Gaussian case. These methods, for different situations, were extended by [6], [7], [8] among others. For a recent review see [9]. We utilize the RKHS theory to prove $X_{\max }$ for both rotation and scale space random fields is indeed the likelihood ratio test statistic. 
The rest of paper is divided into three sections. In Section 1, we introduce the scale and rotation space random fields. In Section 2, we review the main results from RKHS theory and its relation to the covariance function of a random field. Finally, in Section 3, we have the proof for $X_{\max }$ being the likelihood ratio test statistic for testing no signal in rotation and scale space random fields.

\section{Rotation and scale space random fields}

In this section, we briefly introduce two Gaussian random fields extensively used for activation detection in fMRI images; Scale space random field and Rotation space random field.

\subsection{Gaussian scale space random fields}

Let $k$ be an $N$-dimensional kernel such that

$$
\int k^{2}(t) d t=1
$$

[1] define the Gaussian scale space random field as

$$
X(t, \sigma)=\sigma^{-N / 2} \int k\left[\sigma^{-1}(h-t)\right] d Z(h),
$$

where $Z(h)$ is a Gaussian random field defined on a subset of $\mathbb{R}^{N}$ and $\sigma$ is a positive constant.

A justification for working on Gaussian scale space random fields is as follows. Assume the random field $Z(t), t \in \mathbb{R}^{N}$, satisfies

$$
d Z(t)=\xi \sigma_{0}^{-N / 2} k\left[\sigma_{0}^{-1}\left(t-t_{0}\right)\right] d t+d W(t),
$$

where $t_{0} \in T \subset \mathbb{R}^{N}, \xi \geq 0$ and $\sigma_{0}>0$ are fixed values and $W$ is an $N$-dimensional Brownian sheet. The unknown parameter $\theta=\left(\xi, t_{0}, \sigma_{0}\right)$ represents the amplitude, location and scale of a signal added to the noise $d W(t)$. In other words, the shape of the signal in $d Z(t)$ matches the shape of the filter $k$. Models of this form have been used in different scientific contexts. See [2] and references therein. As [10] note, "from a statistical point of view, the scale space field is a continuous wavelet transform of white noise that is designed to be powerful at detecting a localized signal of unknown spatial scale and location."

By replacing $d Z(h)$ from (1.2) in (1.1), the scale space random field takes the form of a signal plus noise model as

$$
X(t, \sigma)=m(t, \sigma ; \theta)+\varepsilon(t, \sigma)
$$

where

$$
m(t, \sigma ; \theta)=\left(\sigma_{0} \sigma\right)^{-1 / 2} \xi \int k\left[\sigma_{0}^{-1 / 2}\left(h-t_{0}\right)\right] k\left[\sigma^{-1 / 2}(h-t)\right] d h
$$

and

$$
\varepsilon(t, \sigma)=\sigma^{-1 / 2} \int k\left[\sigma^{-1 / 2}(h-t)\right] d W(h)
$$


is an $(N+1)$-dimensional zero mean Gaussian random field with the covariance function

$$
R\left[\left(t_{1}, \sigma_{1}\right),\left(t_{2}, \sigma_{2}\right)\right]=\left(\sigma_{1} \sigma_{2}\right)^{-N / 2} \int k\left[\sigma_{1}^{-1}\left(h-t_{1}\right)\right] k\left[\sigma_{2}^{-1}\left(h-t_{2}\right)\right] d h .
$$

See [1]. Note that $m(t, \sigma ; \theta)=\xi R\left[(t, \sigma),\left(t_{0}, \sigma_{0}\right)\right]$.

For testing the hypothesis of no signal, that is, $\xi=0$, [1] consider the test statistic that rejects for large positive values of

$$
X_{\max }=\max _{(t, \sigma) \in T \times\left[\sigma_{1}, \sigma_{2}\right]} X(t, \sigma) .
$$

Then they use two different approaches, the expected Euler characteristic of the excursion set or the volume of tubes, to find an approximate $P$-value.

\subsection{Rotation space random fields}

A possible weakness of the scale space random field is a lack of power to detect signals that are not spherically symmetric. [2] generalize the scale space random field to the rotation space random field as follows.

A Gaussian rotation space random field is defined as

$$
X(t, S)=\operatorname{det}(S)^{-\frac{1}{4}} \int k\left[S^{-\frac{1}{2}}(h-t)\right] d Z(t),
$$

where $k$ is spherically symmetric and $S$ is a $N \times N$ positive definite matrix. The same argument as above for working on the scale space random field justifies working on the rotation space random field. Assume the random field $Z(t), t \in \mathbb{R}^{N}$, satisfies

$$
d Z(t)=\xi \operatorname{det}\left(S_{0}\right)^{-\frac{1}{4}} k\left[S_{0}^{-\frac{1}{2}}\left(t-t_{0}\right)\right] d t+d W(t),
$$

where $S_{0}$ is a member of a fixed parameter set $Q$ of positive definite matrices. The unknown parameter $\theta=\left(\xi, t_{0}, S_{0}\right)$ represents amplitude, location, orientation and scale of signal and $d W(t)$ represents noise.

Similar to the scale space random field, the rotation space random field can be written as

$$
X(t, S)=m(t, S ; \theta)+\varepsilon(t, S),
$$

where

$$
m(t, S ; \theta)=\left(\operatorname{det}\left(S_{0} S\right)\right)^{-\frac{1}{4}} \xi \int k\left[S_{0}^{-\frac{1}{2}}\left(h-t_{0}\right)\right] k\left[S^{-\frac{1}{2}}(h-t)\right] d h,
$$

and

$$
\varepsilon(t, S)=\operatorname{det}(S)^{-\frac{1}{4}} \int k\left[S^{-\frac{1}{2}}(h-t)\right] d W(h)
$$

is an $\left(N+\frac{N(N+1)}{2}\right)$-dimensional zero mean Gaussian random field with the covariance function 


$$
R\left[\left(t_{1}, S_{1}\right),\left(t_{2}, S_{2}\right)\right]=\operatorname{det}\left(S_{1} S_{2}\right)^{-\frac{1}{4}} \int k\left[S_{1}^{-\frac{1}{2}}\left(h-t_{1}\right)\right] k\left[S_{2}^{-\frac{1}{2}}\left(h-t_{2}\right)\right] d h .
$$

See [2]. Again note that

$$
m(t, S ; \theta)=\xi R\left[(t, S),\left(t_{0}, S_{0}\right)\right] .
$$

We use interchangeably $m(t, S ; \theta)$ and $m(t, S)$ for the mean or the signal of the rotation space model.

For testing the hypothesis of no signal, that is, $\xi=0$, [2] consider the test statistic that rejects for large positive values of

$$
X_{\max }=\max _{(t, S) \in T \times Q} X(t, S) .
$$

Then they use two different approaches; the expected Euler characteristic of the excursion set or the volume of tubes, to find an approximate $P$-value for $N=2$, when the kernel has a Gaussian form. [10] use the Gaussian kinematic formula to extend the $P$-value approximation for a general $N$ and any kernel.

For the scale space random field [1] and for the rotation space random field [2], without providing any proof, state that $X_{\max }$ is the likelihood ratio test static for testing the hypothesis of no signal. For the scale space random field, [11] use techniques of Gaussian measures on Hilbert spaces to present a proof. Here we use a reproducing kernel Hilbert space approach to prove $X_{\max }$ is indeed the likelihood ratio test static for both rotation and scale space random fields.

\section{Reproducing kernel Hilbert spaces and random fields}

Reproducing kernel Hilbert spaces arise in different areas of statistics, including statistical signal processing, nonparametric curve estimation, random measures, and limit theorems. In this section, we briefly review the elements of RKHS theory and describe the RKHS generated by the covariance function of a random field.

Let $\mathscr{H}$ be a Hilbert space of real valued functions defined on a set $U$ with the inner product $\langle\cdot, \cdot\rangle_{\mathscr{H}}$ and norm $\|\cdot\|_{\mathscr{H}}$. The real valued function $K(x, y)$, where $x, y \in U$ is called the reproducing kernel of $\mathscr{H}$ if the following conditions hold,

i) for every $y \in U, K_{y}(x)=K(x, y)$ belongs to $\mathscr{H}$, as a function of $x$.

ii) (reproducing property) for every $y \in U$ and $f \in \mathscr{H}, f(y)=\left\langle f, K_{y}\right\rangle \mathscr{H}$.

The Hilbert space $\mathscr{H}$ is called a RKHS if there exists a reproducing kernel for $\mathscr{H}$.

It's clear that a reproducing kernel is a positive semi definite function. The converse of this is a very important theorem for the utility of RKHS in the study of random fields.

Theorem 2.1. (Moore-Aronszajn Theorem) Let $U$ be an index set and $K: U \times U \rightarrow \mathbb{R}$ be a symmetric positive semi definite function. Then there exists a unique Hilbert space of functions on $U$ for which $K$ is a reproducing kernel.

For a proof see [12].

Since the covariance function of a random field is a positive semi definite function by the MooreAronszajn Theorem, there exists a unique RKHS corresponding to a second order random field. In fact, this is a bridge from abstract spaces in mathematics to statistics and gives access to a powerful 
tool for solving statistical signal detection problems. To see that, consider a second-order zero-mean random field $\{X(u), u \in U\}$ with covariance function $K$ and let $L_{X}^{2}$ be the Hilbert space of random variables generated by $X$. Let $\mathscr{H}_{K}$ denote the RKHS with reproducing kernel $K$. Consider the linear map $J$ from $L_{X}^{2}$ to $\mathscr{H}_{K}$ satisfying

$$
J(X(u))=K(u, .), \quad u \in U
$$

The linear map $J$, known as the Loeve-Parzen canonical congruence, establishes an isomorphism between $L_{X}^{2}$ and $\mathscr{H}_{K}$ as stated in the following theorem.

Theorem 2.2. The linear map $J$ is a one-to-one map and for all $\eta_{1}, \eta_{2} \in L_{X}^{2}$ satisfies

$$
\left\langle\eta_{1}, \eta_{2}\right\rangle_{L_{X}^{2}}=\left\langle J\left(\eta_{1}\right), J\left(\eta_{2}\right)\right\rangle_{\mathscr{H}_{K}}
$$

For a proof see [13].

The next step in using the RKHS approach for the problem of signal detection in Gaussian noise is to derive the density of two Gaussian measures with respect to each other if they are equivalent. $[4,5]$ used the RKHS approach to find the likelihood ratio for the detection problem in Gaussian case. These methods were extended for different situations by [6], [7], [8] among the others. For a recent review see [9]. Here we mention a result which suits our models.

Theorem 2.3. Let $U$ be a separable metric space and $\Omega$ be the set of real valued functions defined on $U$, and $\mathscr{F}$ be the sigma field of cylinder sets. Consider a second-order zero-mean Gaussian random field $\{\varepsilon(u), u \in U\}$ with covariance function $K$ and let

$$
X(u)=m(u)+\varepsilon(u)
$$

Let $P_{X}$ and $P_{\varepsilon}$ be two Gaussian probability measures on $(\Omega, \mathscr{F})$ induced by $X(u)$ and $\varepsilon(u)$, respectively. If $m(u) \in \mathscr{H}_{K}$, then $P_{X}$ and $P_{\varepsilon}$ are equivalent and

$$
\frac{d P_{X}}{d P_{\varepsilon}}(x)=\exp \left[J^{-1}(m)-\frac{1}{2}\langle m, m\rangle_{\mathscr{H}_{K}}\right] .
$$

\section{Likelihood ratio test for scale and rotation space random fields}

In this section, we use the RKHS results to derive the likelihood ratio test statistics for scale and rotation space random fields. For this, what we need to do is to find

$$
\lambda(x)=\max _{\theta^{*} \in \Theta} \frac{d P_{\theta^{*}}}{d P_{\theta_{0}}}(x),
$$

where $\frac{d P_{\theta^{*}}}{d P_{\theta_{0}}}(x)$ is the Radon-Nikodym derivative of the measure under the alternative, $P_{\theta^{*}}$, with respect to the measure $P_{\theta_{0}}$ under the null hypothesis of no signal, $\theta=\theta_{0}$. The likelihood ratio test rejects $H_{0}$ for small values of $1 / \lambda(x)$ or equivalently for large values of $\lambda(x)$. 
Theorem 3.1. Let $X(t, S)$ be the Gaussian rotation space random field defined in (1.4). For testing

$$
\left\{\begin{array}{l}
H_{0}: X(t, S)=\varepsilon(t, S) \\
H_{1}: X(t, S)=m(t, S)+\varepsilon(t, S)
\end{array}\right.
$$

the likelihood ratio test statistic is equivalent to

$$
X_{\max }=\max _{(t, S) \in T \times Q} X(t, S) .
$$

Proof. From (1.6), $m(t, S)=\xi R\left[(t, S),\left(t_{0}, S_{0}\right)\right] \in \mathscr{H}_{R}$, where

$$
R\left[\left(t_{1}, S_{1}\right),\left(t_{2}, S_{2}\right)\right]=\operatorname{det}\left(S_{1} S_{2}\right)^{-1 / 4} \int k\left[S_{1}^{-1 / 2}\left(h-t_{1}\right)\right] k\left[S_{2}^{-1 / 2}\left(h-t_{2}\right)\right] d h .
$$

To derive the likelihood ratio, by Theorem 2.3, we need to find $J^{-1}(m)$ and $\langle m, m\rangle_{\mathscr{H}_{R}}=\|m\|_{\mathscr{H}_{R}}$. By the very first definition (2.1) and linearity of $J^{-1}, J^{-1}(m)=\xi x\left(t_{0}, S_{0}\right)$. To find $\|m\|_{\mathscr{C}_{R}}$, note that from the reproducing property and (1.6) we have

$$
\|m\|_{\mathscr{H}_{R}}=\xi^{2}\left\|R\left[.,\left(t_{0}, S_{0}\right)\right]\right\|_{\mathscr{H}_{R}}^{2}=\xi^{2} R\left[\left(t_{0}, S_{0}\right),\left(t_{0}, S_{0}\right)\right] .
$$

Since $R\left[\left(t_{0}, S_{0}\right),\left(t_{0}, S_{0}\right)\right]=\int k^{2}(h) d h=1,\|m\|_{\mathscr{H}_{R}}^{2}=\xi^{2}$. Therefore, the likelihood ratio $\lambda(x)$ becomes

$$
\begin{aligned}
\lambda(x) & =\exp \left[\xi x\left(t_{0}, S_{0}\right)-\frac{\xi^{2}}{2}\right] \\
& =\exp \left\{-\frac{1}{2}\left[\left(\xi-x\left(t_{0}, S_{0}\right)\right)^{2}-x^{2}\left(t_{0}, S_{0}\right]\right\} .\right.
\end{aligned}
$$

Since the $\log$ function is a nondecreasing function, the likelihood ratio test statistic is equivalent to

$$
\max _{\left(t_{0}, S_{0}, \xi\right) \in T \times Q \times(0, \infty)}\left\{-\frac{1}{2}\left[\left(\xi-x\left(t_{0}, S_{0}\right)\right)^{2}-x^{2}\left(t_{0}, S_{0}\right]\right\}\right.
$$

For every fixed value of $\left(t_{0}, S_{0}\right)$ the maximum log likelihood occurs at $\xi=x\left(t_{0}, S_{0}\right)$ and since $\xi \geq 0$, we have

$$
\widehat{\xi}=\left\{\begin{array}{cl}
x\left(\widehat{t_{0}}, \widehat{S_{0}}\right) & \text { if } x\left(\widehat{t_{0}}, \widehat{S_{0}}\right) \geq 0 \\
0 & \text { if } x\left(\widehat{t_{0}}, \widehat{S_{0}}\right)<0
\end{array},\right.
$$

where $x\left(\widehat{t_{0}}, \widehat{S_{0}}\right)=\max _{\left(t_{0}, S_{0}\right) \in T \times Q} x\left(t_{0}, S_{0}\right)$. Therefore, the likelihood ratio test statistic is equivalent to $X_{\max }$.

In a similar manner, we can prove the following theorem for the scale space random field.

Theorem 3.2. Let $X(t, \sigma)$ be the Gaussian scale space random field defined in (1.3). For testing $\xi=0$ against $\xi>0$, the likelihood ratio test statistic is equivalent to

$$
X_{\max }=\max _{(t, \sigma) \in T \times\left[\sigma_{1}, \sigma_{2}\right]} X(t, \sigma) .
$$




\section{Discussion}

In this paper, we proved that for both rotation and scale space random fields the global maximum of the image is indeed the likelihood ratio test statistic. The result can be extended to some other random fields beyond these two random fields. As long as we know how to find $J^{-1}(m)$ and $\|m\|$, we can find the likelihood ratio test statistic. It is not very obvious to the authors how to find $J^{-1}(m)$ and $\|m\|$ for any given random field. But it is not very hard to extend the result to the case that the random field $Z(t)$, for $t \in \mathbb{R}^{N}$, defined as

$$
d Z(t)=\sum_{l=1}^{L} \xi_{i} \sigma_{0 l}^{-N / 2} k\left[\sigma_{0 l}^{-1}\left(t-t_{l 0}\right)\right] d t+d W(t),
$$

where $t_{l 0} \in T \subset \mathbb{R}^{N}, \xi_{l}$, and $\sigma_{l 0}>0, l=1, \cdots, L$ are fixed values and $W$ is an $N$-dimensional Brownian sheet. The unknown parameter $\theta_{l}=\left(\xi, t_{0 l}, \sigma_{0 l}\right)$ represents the amplitude, location and scale of the $l$-th signal added to the noise $d W(t)$.

This case and some other cases along with the approximate distribution of the likelihood ratio test statistic is an undergoing work and will be presented in a future publication.

\section{Acknowledgement}

The authors would like to thank the two anonymous referees for the critical comments and suggestions which helped to simplify the proof and improve the quality of the paper.

\section{References}

[1] D. O. Siegmund and K. J. Worsley, Testing for a signal with unknown location and scale in a stationary Gaussian random field, The Annals of Statistics 23(2) (1995) 608-639.

[2] K. Shafie, B. Sigal, D. Siegmund and K. Worsley, Rotation space random fields with an application to fMRI data, Annals of Statistics 31(6) (2003) 1732-1771.

[3] M. R. F. Rohani, Bayesian approach to the scale space random fields, $\mathrm{PhD}$ thesis, Shahid Beheshti University, Tehran, Iran2006.

[4] E. Parzen, An approach to time series analysis, The Annals of Mathematical Statistics 32 (1961) 951989.

[5] E. Parzen, Probability density functionals and reproducing kernel Hilbert spaces, in Proceedings of the Symposium on Time Series Analysis, 196, Wiley, New York1963, pp. 155-169.

[6] T. Kailath and H. L. Weinert, An RKHS approach to detection and estimation problems- part i: Deterministic signals in Gaussian noise, IEEE Transaction on Information Theory IT-17 (1971) 530-549.

[7] T. Kailath and H. L. Weinert, An RKHS approach to detection and estimation problems- part ii: Gaussian signal detection, IEEE Transaction on Information Theory IT-22(1) (1975) 15-23.

[8] D. L. Duttweiler and T. Kailath, RKHS approach to detection and estimation problems-v: Parameter estimation, Information Theory, IEEE Transactions on 19(1) (1973) 29-37.

[9] T. Kailath and H. V. Poor, Detection of stochastic processes, Information Theory, IEEE Transactions on 44(6) (1998) 2230-2231.

[10] R. J. Adler, E. Subag, J. E. Taylor et al., Rotation and scale space random fields and the Gaussian kinematic formula, The Annals of Statistics 40(6) (2012) 2910-2942.

[11] M. R. F. Rohani, K. Shafie and S. Noorbaloochi, A Bayesian signal detection procedure for scale-space random fields, The Canadian Journal of Statistics 34(2) (2006) 311-325.

[12] N. Aronszajn, Theory of reproducing kernels, Transactions of the American Mathematical Society 68(3) (1950) 337-404.

[13] A. Berlinet and C. Thomas-Agnan, Reproducing kernel Hilbert spaces in probability and statistics (Springer, 2004). 